\title{
Long Interspersed Element
}

National Cancer Institute

\section{Source}

National Cancer Institute. Long Interspersed Element. NCI Thesaurus. Code C13803.

A highly repetitive retrotransposon-like sequence that is $6000-8000$ base pairs in length. It

may contain an RNA polymerase II promoter domain, repetitive target sequences, and open reading frames encoding proteins with endonuclease and reverse transcriptase activity. 